\title{
Preparation of C-3,5-Acyl Furanoses via Highly Selective Intramolecular Acyl Migration
}

\author{
Feng Xu*, Bryon Simmons, Kimberly Savary, Chunhua Yang, and Robert A. Reamer, \\ Department of Process Research, Merck Research Laboratories, \\ Rahway, NJ 07065, USA \\ feng_xu@merck.com
}

\section{Supporting Information}

$\underline{\text { Table of Contents }}$

1. Method for HRMS

Page S2

2. Through process procedures for preparation of $\mathbf{4 a}$

Page S2

3. Analytical data for $\mathbf{4 a - i}, \mathbf{1 0}, \mathbf{1 2}$

Page S2 -S7

4. General procedure for acyl migration

Page S7

5. Preparation of $6 \mathbf{a}$

Page S7

6. Analytical data for $\mathbf{6 a - i}, \mathbf{1 1}, \mathbf{1 3}$

Page S7-S12

7. Preparation of 8

Page S12 
Method for HRMS: High resolution mass spectroscopy (HRMS) measurements were acquired on Micromass Ultima US Quadrupole Time of Flight (QTof) mass spectrometer. Elctrospray ionization was used in the negative ion mode. Samples were dissolved in $\mathrm{MeCN}$ and injected into a carrier composed of 50:50 water/MeCN with 0.02\% HOAc. Coumpounds were measured as deprotonated molecular ions, $\mathrm{M}-\mathrm{H}$, deprotonated water adducts, $\mathrm{M}-\mathrm{H}+\mathrm{H}_{2} \mathrm{O}$ or acetate adducts, $\mathrm{M}+\mathrm{CH}_{3} \mathrm{COO}^{-}$. The internal lock mass was raffinose. Mass calibration was performed using $\mathrm{NaOAc}$ as the calibrant. The mass spectrometer was preformed at a resolution $(\mathrm{M} / \Delta \mathrm{M})$ of 10,000 .

\section{Through process procedures for preparation of $4 a$}

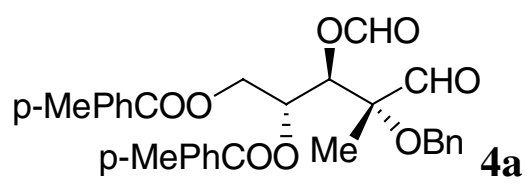

To a yellow solution of $1(100 \mathrm{~g}, 0.308 \mathrm{~mol})$ and pyridine $(74.4 \mathrm{~mL}, 0.924 \mathrm{~mol})$ in $700 \mathrm{~mL}$ of $\mathrm{MeCN}$ at ambient temperature was added p-toluoyl chloride $(92 \mathrm{~mL}, 0.6776$ mol) dropwise over $30 \mathrm{~min}$. During the addition, the reaction temperature was controlled below $45{ }^{\circ} \mathrm{C}$ by using external cooling. The reaction was aged at $50{ }^{\circ} \mathrm{C}$ for $16 \mathrm{~h}$ to achieve $>99 \%$ conversion. Then, $\mathrm{HClO}_{4}(60 \%, 60 \mathrm{~mL}, 0.924 \mathrm{~mol})$ and $\mathrm{H}_{2} \mathrm{O}(250 \mathrm{~mL})$ were added. After $10 \mathrm{~h}$ age at $55^{\circ} \mathrm{C}$, the reaction solution was allowed to cool to $0-5{ }^{\circ} \mathrm{C}$. A solution of periodic acid ( $86 \mathrm{~g}, 0.3696 \mathrm{~mol})$ in $200 \mathrm{~mL}$ of $\mathrm{H}_{2} \mathrm{O}$ was added dropwise over 1-2 $\mathrm{h}$. The reaction was further aged for 30 minutes at $0-5{ }^{\circ} \mathrm{C}$. iPrOAc $(500 \mathrm{~mL})$ and $\mathrm{H}_{2} \mathrm{O}(200 \mathrm{~mL})$ were added. The organic phase was separated and washed with 500 $\mathrm{mL}$ of $\mathrm{H}_{2} \mathrm{O}, 400 \mathrm{~mL}$ of half saturated $\mathrm{NaHCO}_{3}, 300 \mathrm{~mL}$ of $5 \%$ sodium thiosulfate and $5 \%$ $\mathrm{NaCl}$ solution. The organic phase was concentrated in vacuo to give the crude product in about $150 \mathrm{~mL}$ in iPrOAc, while $\mathrm{MeCN}$ was also removed during the concentration. This crude product can be used directly for acyl migration (see procedure B for the preparation of $\mathbf{6 a}$ ). $\mathbf{4 a}: \mathbf{5}$ is typically about 9:1. Three-step assay yield for $\mathbf{4 a}$ and $\mathbf{5}$ is $88 \%$.

4a could also be purified through $\mathrm{SiO}_{2}$ column chromatography eluted with EtOAc/hexane (25:75). $\quad$ 1H NMR $\left(\mathrm{CDCl}_{3}, 400 \mathrm{MHz}\right): \delta 9.57$ (s, $\left.1 \mathrm{H}\right), 8.23(\mathrm{~d}, J=0.6$ Hz, $1 \mathrm{H}$ ), 7.89 (d, $J=8.2 \mathrm{~Hz}, 2 \mathrm{H}), 7.84$ (d, $J=8.27 \mathrm{~Hz}, 2 \mathrm{H}), 7.33$ (m, $4 \mathrm{H}), 7.29$ (m, 1 H), $7.24(\mathrm{~d}, J=8.2 \mathrm{~Hz}, 2 \mathrm{H}), 7.23(\mathrm{~d}, J=8.1 \mathrm{~Hz}, 2 \mathrm{H}), 5.90(\mathrm{dd}, J=0.6,8.2 \mathrm{~Hz}, 1 \mathrm{H})$, 
$5.84(\mathrm{ddd}, J=2.4,4.5,8.2 \mathrm{~Hz}, 1 \mathrm{H}), 4.76(\mathrm{dd}, J=2.4,12.6 \mathrm{hz}, 1 \mathrm{H}), 4.72(\mathrm{~d}, J=11.4 \mathrm{~Hz}$, $1 \mathrm{H}), 4.62(\mathrm{~d}, J=11.4 \mathrm{~Hz}, 1 \mathrm{H}), 4.42(\mathrm{dd}, J=4.5,12.6 \mathrm{~Hz}, 1 \mathrm{H}), 2.41$ (s, $3 \mathrm{H}), 2.40$ (s, 3 $\mathrm{H}), 1.45$ (s, $3 \mathrm{H})$.

${ }^{13} \mathrm{C} \mathrm{NMR}\left(\mathrm{CDCl}_{3}, 100 \mathrm{MHz}\right): \delta 202.1,166.3,165.0,159.3,144.5,144.1,137.7,130.2$, $130.0,129.5,129.4,128.7,128.0,127.5,127.0,126.6,83.3,73.8,69.3,67.0,62.6,21.9$, 16.5 .

HRMS: Calcd for $\mathrm{C}_{30} \mathrm{H}_{30} \mathrm{O}_{8}-\mathrm{H}+\mathrm{H}_{2} \mathrm{O}$ m/z 535.1968; Found 535.1953.

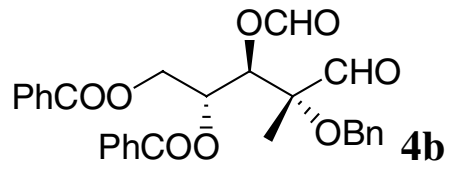

1H NMR (CDCl3, $400 \mathrm{MHz}): \delta 9.58(\mathrm{~s}, 1 \mathrm{H}), 8.25$ (s, $1 \mathrm{H}), 8.01(\mathrm{~m}, 2 \mathrm{H}), 7.95(\mathrm{~m}, 2 \mathrm{H})$, 7.56 (m, 2 H), 7.46 (m, 4 H), 7.2 (m, 5 H), 5.93 (d, J=8.9 Hz, 1 H), 5.86 (ddd, $J=2.4$, 4.6, 8.9 Hz, $1 \mathrm{H}), 4.81(\mathrm{dd}, J=2.4,12.7 \mathrm{~Hz}, 1 \mathrm{H}), 4.72(\mathrm{~d}, J=11.3 \mathrm{~Hz}, 1 \mathrm{H}), 4.62(\mathrm{~d}, J=$ $11.3 \mathrm{~Hz}, 1 \mathrm{H}), 4.45$ (dd, $J=4.6,12.7 \mathrm{~Hz}, 1 \mathrm{H}), 1.46$ (s, $3 \mathrm{H})$.

${ }^{13} \mathrm{C}$ NMR $\left(\mathrm{CDCl}_{3}, 100 \mathrm{MHz}\right): \delta 202.1,166.3,164.9,159.3,137.6,133.7,133.4,130.1$, $130.0,129.7,129.2,128.8,128.7,128.1,127.5,83.3,73.6,69.5,67.0,62.7,16.4$.

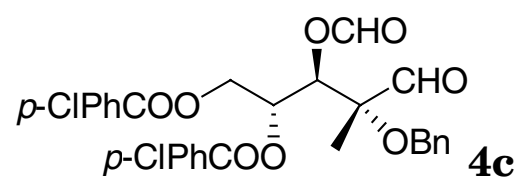

4c plus about 10\% deformylated product: $82 \%$ isolated yield for three-step through process.

1H NMR (CDCl3, $400 \mathrm{MHz}): \delta 9.58(\mathrm{~s}, 1 \mathrm{H}), 8.25(\mathrm{~s}, 1 \mathrm{H}), 7.94(\mathrm{~d}, J=8.5 \mathrm{~Hz}, 2 \mathrm{H})$, $7.85(\mathrm{~d}, J=8.5 \mathrm{~Hz}, 2 \mathrm{H}), 7.42(\mathrm{~d}, J=8.5 \mathrm{~Hz}, 2 \mathrm{H}), 7.41(\mathrm{~d}, J=8.5 \mathrm{~Hz}, 2 \mathrm{H}), 7.34$ (m, 5 H), $5.90(\mathrm{~d}, J=8.3 \mathrm{~Hz}, 1 \mathrm{H}), 5.82(\mathrm{ddd}, J=2.3,4.7,8.3 \mathrm{~Hz}, 1 \mathrm{H}), 4.82(\mathrm{dd}, J=2.3,12.6$ $\mathrm{Hz}, 1 \mathrm{H}), 4.72(\mathrm{~d}, J=11.3 \mathrm{~Hz}, 1 \mathrm{H}), 4.62(\mathrm{~d}, J=11.3 \mathrm{~Hz}, 1 \mathrm{H}), 4.42(\mathrm{dd}, J=4.7,12.6$ $\mathrm{Hz}, 1 \mathrm{H}), 1.46$ (s, $3 \mathrm{H})$.

${ }^{13} \mathrm{C} \mathrm{NMR}\left(\mathrm{CDCl}_{3}, 100 \mathrm{MHz}\right): \delta$ 201.9, 165.4, 164.1, 159.3, 140.4, 140.0, 137.5, 131.4, $131.3,129.2,129.1,128.7,128.1,128.0,127.6,127.5,83.2,73.3,69.7,67.1,62.8,16.3$. HRMS: Calcd for $\mathrm{C}_{28} \mathrm{H}_{24} \mathrm{Cl}_{2} \mathrm{O}_{8}+\mathrm{Ac}^{-} \mathrm{m} / \mathrm{z}$ 617.0981; Found 617.0961. 


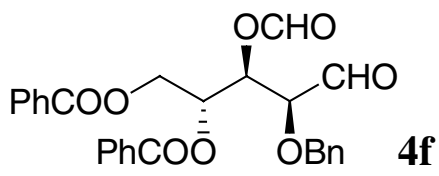

4f plus about 5\% deformylated product: $85 \%$ isolated yield for three-step through process.

${ }^{1} \mathrm{H}$ NMR $\left(\mathrm{CDCl}_{3}, 400 \mathrm{MHz}\right): \delta 9.69$ (s, $\left.1 \mathrm{H}\right), 8.10$ (s, $\left.1 \mathrm{H}\right), 7.99$ (m, $\left.2 \mathrm{H}\right), 7.96(\mathrm{~m}, 2 \mathrm{H})$, $7.56(\mathrm{~m}, 2 \mathrm{H}), 7.44(\mathrm{~m}, 4 \mathrm{H}), 7.28(\mathrm{~m}, 2 \mathrm{H}), 7.23(\mathrm{~m}, 3 \mathrm{H}), 5.86(\mathrm{ddd}, J=0.8,2.8,7.6 \mathrm{~Hz}$, $1 \mathrm{H}), 5.79$ (ddd, $J=2.7,4.7,7.6 \mathrm{~Hz}, 1 \mathrm{H}), 4.88$ (dd, $J=2.7,12.7 \mathrm{~Hz}, 1 \mathrm{H}), 4.76$ (d, $J=$ $11.4 \mathrm{~Hz}, 1 \mathrm{H}), 4.57$ (d, $J=11.4 \mathrm{~Hz}, 1 \mathrm{H}), 4.52$ (dd, $J=4.7,12.7 \mathrm{~Hz}, 1 \mathrm{H}), 4.15(\mathrm{~m}, 1 \mathrm{H})$. ${ }^{13} \mathrm{C} \mathrm{NMR}\left(\mathrm{CDCl}_{3}, 100 \mathrm{MHz}\right): \delta$ 199.6, 166.2, 165.3, 159.5, 136.0, 133.8, 133.5, 130.1, $129.9,129.6,129.2,128.9,128.84,128.81,128.73,128.71,81.1,73.9,69.7,69.3,62.3$.

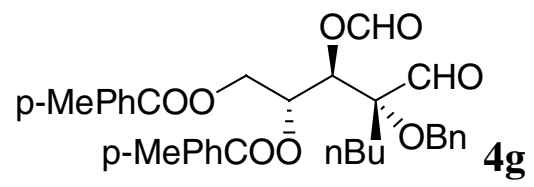

${ }^{1} \mathrm{H}$ NMR $\left(\mathrm{CDCl}_{3}, 400 \mathrm{MHz}\right): \delta 9.48(\mathrm{~s}, 1 \mathrm{H}), 8.30(\mathrm{~s}, 1 \mathrm{H}), 7.95(\mathrm{~d}, J=8.0 \mathrm{~Hz}, 2 \mathrm{H})$, $7.88(\mathrm{~d}, J=8.4 \mathrm{~Hz}, 2 \mathrm{H}), 7.42(\mathrm{~m}, 4 \mathrm{H}), 7.35(\mathrm{~m}, 1 \mathrm{H}), 7.25(\mathrm{~m}, 4 \mathrm{H}), 6.07$ (d, J = 9.6 Hz, $1 \mathrm{H}), 5.78$ (ddd, $J=2.0,4.4,9.6 \mathrm{~Hz}, 1 \mathrm{H}), 4.92$ (d, $J=11.2 \mathrm{~Hz}, 1 \mathrm{H}), 4.78$ (dd, $J=2.0$, $12.5 \mathrm{~Hz}, 1 \mathrm{H}), 4.68$ (d, J=11.2 Hz, $1 \mathrm{H}), 4.40$ (dd, $J=4.4,12.5 \mathrm{~Hz}, 1 \mathrm{H}), 2.41$ (s, $3 \mathrm{H})$, $2.40(\mathrm{~s}, 3 \mathrm{H}), 2.06(\mathrm{~m}, 1 \mathrm{H}), 1.79(\mathrm{~m}, 1 \mathrm{H}), 1.39$ (m, $1 \mathrm{H}), 1.29(\mathrm{~m}, 2 \mathrm{H}), 1.20(\mathrm{~m}, 1 \mathrm{H})$, $0.88(\mathrm{t}, J=7.2 \mathrm{~Hz}, 3 \mathrm{H})$.

${ }^{13} \mathrm{C} \mathrm{NMR}\left(\mathrm{CDCl}_{3}, 100 \mathrm{MHz}\right): \delta 203.7,166.1,164.5,144.4,143.9,137.5,129.9,129.8$, $129.3,129.2,128.5,127.8,127.6,126.8,126.3,85.0,74.5,68.9,66.2,62.1,30.5,24.5$, 23.1, 21.7, 13.8 .

HRMS: Calcd for $\mathrm{C}_{33} \mathrm{H}_{36} \mathrm{O}_{8}+\mathrm{Ac}^{-} \mathrm{m} / \mathrm{z}$ 619.2543; Found 619.2512.

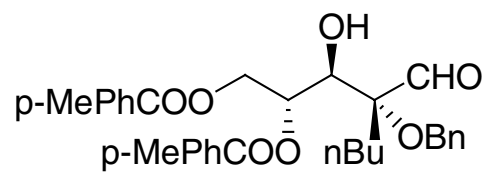


${ }^{1} \mathrm{H}$ NMR ( $\left.\mathrm{CDCl}_{3}, 400 \mathrm{MHz}\right): \delta 9.49$ (s, $\left.1 \mathrm{H}\right), 7.90$ (d, $\left.J=8.1 \mathrm{~Hz}, 2 \mathrm{H}\right), 7.87$ (d, $J=8.2$ $\mathrm{Hz}, 2 \mathrm{H}), 7.37$ (m, 4 H), 7.26 (m, 5 H), 5.50 (ddd, $J=2.8,2.8,9.4$ Hz, $1 \mathrm{H}), 4.94$ (dd, $J=$ $3.1,12.6 \mathrm{~Hz}, 1 \mathrm{H}), 4.87(\mathrm{~d}, J=11.4 \mathrm{~Hz}, 1 \mathrm{H}), 4.69(\mathrm{~d}, J=11.4 \mathrm{~Hz}, 1 \mathrm{H}), 4.61(\mathrm{dd}, J=$ 2.5, $12.6 \mathrm{~Hz}, 1 \mathrm{H}), 4.19$ (dd, $J=6.0,9.4 \mathrm{~Hz}, 1 \mathrm{H}), 3.41(\mathrm{~d}, J=6.0 \mathrm{~Hz}, 1 \mathrm{H}), 2.42(\mathrm{~s}, 3 \mathrm{H})$, 2.41 (s, $3 \mathrm{H}), 2.32(\mathrm{~m}, 1 \mathrm{H}), 1.82(\mathrm{~m}, 1 \mathrm{H}), 1.49-1.20(\mathrm{~m}, 4 \mathrm{H}), 0.88$ (t, $J=7.1 \mathrm{~Hz}, 3 \mathrm{H})$. ${ }^{13} \mathrm{C}$ NMR $\left(\mathrm{CDCl}_{3}, 100 \mathrm{MHz}\right): \delta 205.5,167.8,165.0,144.45,144.37,138.3,130.14$, $130.12,129.5,129.4,128.6,127.8,127.7,126.82,126.79,86.0,74.3,71.6,66.6,63.4$, 30.9, 25.1, 23.5, 21.9, 14.0.

HRMS: Calcd for $\mathrm{C}_{32} \mathrm{H}_{36} \mathrm{O}_{7}+\mathrm{Ac}^{-} \mathrm{m} / \mathrm{z}$ 591.2600; Found 591.2577.

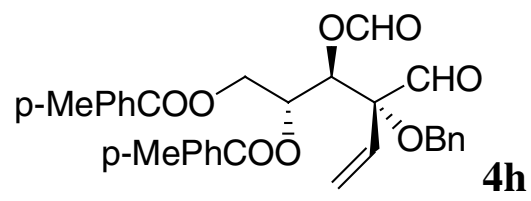

${ }^{1} \mathrm{H}$ NMR (CDCl $\left.3,400 \mathrm{MHz}\right): \delta 9.51(\mathrm{~s}, 1 \mathrm{H}), 8.17(\mathrm{~d}, J=0.6 \mathrm{~Hz}, 1 \mathrm{H}), 7.90(\mathrm{~d}, J=8.2$ Hz, 2H), 7.85 (d, J = 8.2 Hz, 2 H), 7.33 (m, 4 H), 7.29 (m, 2 H), 7.23 (m, 4 H), 6.12 (dd, $J=0.6,8.0 \mathrm{~Hz}, 1 \mathrm{H}), 5.90(\mathrm{dd}, J=11.1,17.7 \mathrm{~Hz}, 1 \mathrm{H}), 5.87$ (m, $1 \mathrm{H}), 5.59$ (d, $J=11.1$ $\mathrm{Hz}, 1 \mathrm{H}), 5.55(\mathrm{~d}, J=17.7 \mathrm{~Hz}, 1 \mathrm{H}), 4.76(\mathrm{dd}, J=2.4,12.5 \mathrm{~Hz}, 1 \mathrm{H}), 4.75(\mathrm{~d}, J=11.6$ $\mathrm{Hz}, 1 \mathrm{H}), 4.73$ (d, $J=11.6 \mathrm{~Hz}, 1 \mathrm{H}), 4.43$ (dd, $J=5.2,12.5 \mathrm{~Hz}, 1 \mathrm{H}), 2.4(\mathrm{~s}, 6 \mathrm{H})$. ${ }^{13} \mathrm{C} \mathrm{NMR}\left(\mathrm{CDCl}_{3}, 100 \mathrm{MHz}\right): \delta 198.2,166.1,164.5,159.0,144.2,143.8,137.4,130.4$, $129.8,129.7,129.2,129.0,128.4,127.7,127.2,126.7,126.4,121.6,86.6,72.7,69.0$, 67.4, 62.3, 21.6.

HRMS: Calcd for $\mathrm{C}_{31} \mathrm{H}_{30} \mathrm{O}_{8}+\mathrm{Ac}^{-} \mathrm{m} / \mathrm{z}$ 589.2074; Found 589.2079.

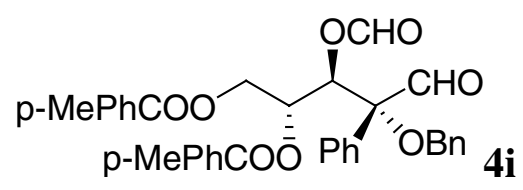

${ }^{1} \mathrm{H} \mathrm{NMR}\left(\mathrm{CDCl}_{3}, 400 \mathrm{MHz}\right): \delta 9.68(\mathrm{~s}, 1 \mathrm{H}), 7.99(\mathrm{~d}, J=0.7 \mathrm{~Hz}, 1 \mathrm{H}), 7.90(\mathrm{~d}, J=8.2$ Hz, 2 H), 7.85 (d, $J=8.2$ Hz, 2 H), 7.49 (m, 2 H), 7.37 (m, 8 H), 7.24 (m, 4 H), 6.47 (d, $J$ $=7.6 \mathrm{hz}, 1 \mathrm{H}), 5.95(\mathrm{ddd}, J=2.6,5.3,7.6 \mathrm{~Hz}, 1 \mathrm{H}), 4.78(\mathrm{~d}, J=11.7 \mathrm{~Hz}, 1 \mathrm{H}), 4.72(\mathrm{~d}, J$ 
$=11.7 \mathrm{~Hz}, 1 \mathrm{H}), 4.63(\mathrm{dd}, J=2.6,12.5 \mathrm{~Hz}, 1 \mathrm{H}), 4.36(\mathrm{dd}, J=5.3,12.5 \mathrm{~Hz}, 1 \mathrm{H}), 2.40(\mathrm{~s}$, $6 \mathrm{H})$.

${ }^{13} \mathrm{C} \mathrm{NMR}\left(\mathrm{CDCl}_{3}, 100 \mathrm{MHz}\right): \delta 197.9,166.3,165.0,159.1,144.4,144.0,137.6,133.0$, 130.2, 130.0, 129.5, 129.4, 129.27, 129.23, 128.7, 128.1, 127.8, 127.6, 127.1, 126.6, 87.4, $73.3,69.5,68.0,62.8,21.9$.

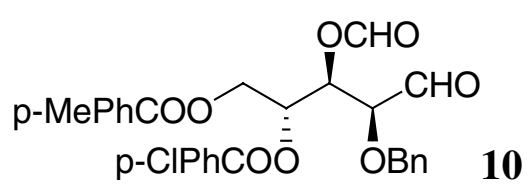

10 plus about $15 \%$ deformylated product: $94 \%$ isolated yield for three-step through process.

${ }^{1} \mathrm{H} \mathrm{NMR}\left(\mathrm{CDCl}_{3}, 400 \mathrm{MHz}\right): \delta 9.81$ (s, $\left.1 \mathrm{H}\right), 8.10$ (s, $\left.1 \mathrm{H}\right), 7.86(\mathrm{~m}, 4 \mathrm{H}), 7.40$ (d, $J=8.7$ $\mathrm{Hz}, 2 \mathrm{H}), 7.26$ (m, 7H), 5.83 (dd, $J=2.9,7.2 \mathrm{~Hz}, 1 \mathrm{H}), 5.74(\mathrm{~m}, 1 \mathrm{H}), 4.87$ (dd, $J=2.6$, $12.7 \mathrm{~Hz}, 1 \mathrm{H}), 4.75(\mathrm{~d}, J=11.6 \mathrm{~Hz}, 1 \mathrm{H}), 4.56(\mathrm{~d}, J=11.6 \mathrm{~Hz}, 1 \mathrm{H}), 4.48(\mathrm{dd}, J=4.8$, $12.7 \mathrm{~Hz}, 1 \mathrm{H}), 4.13(\mathrm{~d}, J=4.2 \mathrm{~Hz}, 1 \mathrm{H}), 2.41(\mathrm{~s}, 3 \mathrm{H})$.

${ }^{13} \mathrm{C}$ NMR (CDCl3, $\left.100 \mathrm{MHz}\right): \delta 199.6,166.2,164.5,159.4,144.3,140.4,136.0,131.4$, 127.0, 129.4, 129.1, 128.9, 128.8, 128.7, 127.7, 126.8, 81.0, 73.9, 70.1, 69.3, 21.9.

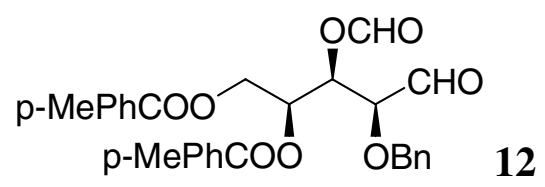

12 plus about $15 \%$ deformylated product was isolated.

${ }^{1} \mathrm{H}$ NMR (CDCl3, $\left.400 \mathrm{MHz}\right): \delta 9.63$ (s, $1 \mathrm{H}$ ), 8.09 (s, $1 \mathrm{H}$ ), 7.88 (m, $\left.4 \mathrm{H}\right), 7.60$ - 7.20 (m, 9 H), $5.85(\mathrm{dd}, J=5.2,10.0 \mathrm{~Hz}, 1 \mathrm{H}), 5.78(\mathrm{~m}, 1 \mathrm{H}), 4.82(\mathrm{~d}, J=11.7 \mathrm{~Hz}, 1 \mathrm{H}), 4.63(\mathrm{~d}$, $J=11.7 \mathrm{~Hz}, 1 \mathrm{H}), 4.53(\mathrm{dd}, J=4.4,12.0 \mathrm{~Hz}, 1 \mathrm{H}), 4.39(\mathrm{dd}, J=5.61,12.0 \mathrm{~Hz}, 1 \mathrm{H})$, $4.18(\mathrm{~m}, 1 \mathrm{H}), 2.40(\mathrm{~s}, 6 \mathrm{H})$.

${ }^{13} \mathrm{C}$ NMR (CDCl3, $\left.100 \mathrm{MHz}\right): \delta 199.4,165.9,165.1,159.5,144.3,143.9,136.0,129.9$, $129.7,129.2,129.1,128.7,128.6,128.4,128.3,126.2,80.1,73.5,69.8,69.2,63.8,22.6$, 22.5. 


\section{General procedure for acyl migration}

To a solution of 4a-h (1 equiv) in an alcohol solvent $(10-20 \mathrm{~mL} / \mathrm{g})$ under $\mathrm{N}_{2}$ was added $\mathrm{iPr}_{2} \mathrm{NH}$ (1.5 equiv.) at $0-5{ }^{\circ} \mathrm{C}$. The reaction solution was aged at specified temperature till the reaction was completed. The residue upon concentration in vacuo was purified on column chromatography to afford the furanoses and pyranoses.

\section{Preparation of $6 a$}

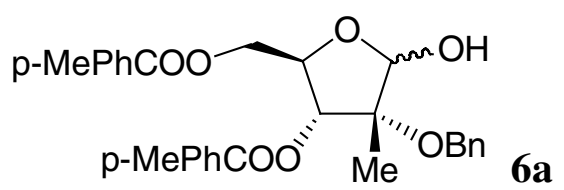

Procedure A: A solution of $4 \mathbf{a}(1.0 \mathrm{~g}, 1.98 \mathrm{mmol})$ and $\mathrm{iPr}_{2} \mathrm{NH}(300 \mathrm{mg}, 2.97 \mathrm{mmol})$ in $\mathrm{MeOH}(10 \mathrm{~mL})$ was aged at $0-5{ }^{\circ} \mathrm{C}$ for 1 day. The crude was purified on $\mathrm{SiO}_{2}$ column eluted with iPrOAc/hexane (3:7) to give $\mathbf{6 a}(840 \mathrm{mg}, 86 \%$ yield).

Procedure B: A solution of $\mathbf{4 a}$ and $\mathbf{5}(\mathbf{4 a : 5}=\mathrm{ca} .9: 1)$ in iPrOAc, obtained from a through process (started with $0.154 \mathrm{~mol}$ of $\mathbf{1}$ ), was concentrated in vacuo to about $150 \mathrm{ml}$. $\mathrm{MeOH}$ (400 mL) was added and the solution was cooled to $0{ }^{\circ} \mathrm{C}$ and diisoproylamine $(7.8 \mathrm{~g}$, $0.077 \mathrm{~mol}$ ) was added over $10 \mathrm{~min}$. The solution was aged at $0^{\circ} \mathrm{C}$ for 2 days. Then, $2 \mathrm{~N}$ $\mathrm{HCl}(160 \mathrm{~mL})$ was added dropwise at 0 to $10{ }^{\circ} \mathrm{C}$. iPrOAc $(500 \mathrm{~mL})$ and $250 \mathrm{~mL}$ of water were added and the organic phase was separated. The organic phase was washed with $150 \mathrm{~mL}$ of $10 \% \mathrm{NaHCO}_{3}$, followed by $150 \mathrm{~mL}$ of $10 \% \mathrm{NaCl}$. Then, the organic phase was concentrated in vacuo to about $300 \mathrm{~mL}$. By HPLC: $>98$ A\% conversion and $96 \%$ selectivity (6a vs. 7a). $84 \%$ assay yield (63. $4 \mathrm{~g}$ of $\mathbf{6 a}$, four steps starting from 1a).

${ }^{1} \mathrm{H} \mathrm{NMR}\left(\mathrm{CDCl}_{3}, 400 \mathrm{MHz}\right): \delta$ The ratio of $\alpha: \beta$ isomers in $\mathrm{CDCl}_{3}$ is about 5 to 2 . For the $\alpha$ isomer: $\delta 7.92(\mathrm{~d}, J=8.1 \mathrm{~Hz}, 2 \mathrm{H}), 7.88(\mathrm{~d}, J=8.8 \mathrm{~Hz}, 2 \mathrm{H}), 7.39-7.18(\mathrm{~m}, 9 \mathrm{H})$, $5.34(\mathrm{~d}, J=5.9 \mathrm{~Hz}, 1 \mathrm{H}), 5.21(\mathrm{~s}, 1 \mathrm{H}), 4.73(\mathrm{~d}, J=11.0,1 \mathrm{H}), 4.70-4.62(\mathrm{~m}, 2 \mathrm{H}), 4.61$ $(\mathrm{d}, J=11.0 \mathrm{~Hz}, 1 \mathrm{H}), 4.52(\mathrm{dd}, J=5.1,11.7 \mathrm{~Hz}, 1 \mathrm{H}), 4.20(\mathrm{~s}, \mathrm{br}, 1 \mathrm{H}), 2.42(\mathrm{~s}, 3 \mathrm{H})$, $2.40(\mathrm{~s}, 3 \mathrm{H}), 1.63(\mathrm{~s}, 3 \mathrm{H})$; for the $\alpha$ isomer: $\delta 7.95-7.87(\mathrm{~m} 4 \mathrm{H}), 7.38-7.16(\mathrm{~m}, 9 \mathrm{H})$, $5.66(\mathrm{~d}, J=7.3 \mathrm{~Hz}, 1 \mathrm{H}), 5.36(\mathrm{~s}, 1 \mathrm{H}), 4.77(\mathrm{~d}, J=11.7 \mathrm{~Hz}, 1 \mathrm{H}), 4.70-4.62(\mathrm{~m}, 3 \mathrm{H})$, 4.51 (m, 1 H), 4.20 (s, br, 1 H), 2.42 (s, 3 H), 2.38 (s, 3 H), 1.55 (s, 3 H). 
${ }^{13} \mathrm{C} \mathrm{NMR}\left(\mathrm{CDCl}_{3}, 100 \mathrm{MHz}\right): \delta 166.7,166.5,166.3,166.1,144.6,144.4,144.0,143.9$, $137.8,130.2,130.1,130.0,129.4,129.38,129.3,128.6,128.5,128.0,127.5,127.46$, 127.3, 127.2, 127.1, 126.8, 126.5, 101.5, 101.3, 83.9, 80.6, 79.2, 78.6, 77.4, 76.9, 67.4, $66.2,65.3,64.2,21.9,21.78,19.4,15.8$.

HRMS: Calcd for $\mathrm{C}_{29} \mathrm{H}_{30} \mathrm{O}_{7}-\mathrm{H} \mathrm{m} / \mathrm{z} 489.1913$; Found 489.1916.

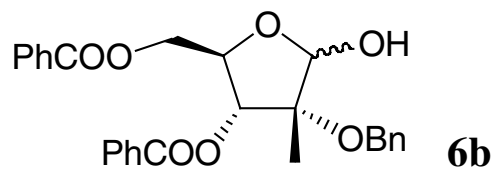

${ }^{1} \mathrm{H}$ NMR $\left(\mathrm{CDCl}_{3}, 400 \mathrm{MHz}\right)$ : The ratio of $\alpha: \beta$ isomers in $\mathrm{CDCl}_{3}$ is about 10 to 6 . For the $\alpha$ isomer: $\delta$ 8.05-7.98 (m, 4H), $7.60-7.29(\mathrm{~m}, 11 \mathrm{H}), 5.36(\mathrm{~d}, J=6.0 \mathrm{~Hz}, 1 \mathrm{H}), 5.22$ (s, $1 \mathrm{H}), 4.72(\mathrm{~d}, J=11.2 \mathrm{~Hz}, 1 \mathrm{H}), 4.73-4.64(\mathrm{~m}, 2 \mathrm{H}), 4.62(\mathrm{~d}, J=11.2 \mathrm{~Hz}, 1 \mathrm{H}), 4.55$ $(\mathrm{dd}, J=4.8,11.6 \mathrm{~Hz}, 1 \mathrm{H}), 4.12(\mathrm{br} \mathrm{s}, 1 \mathrm{H}), 1.65(\mathrm{~s}, 3 \mathrm{H})$; for the $\beta$ isomer: $\delta$ 8.05-7.98 (m, 4H), $7.60-7.29(\mathrm{~m}, 11 \mathrm{H}), 5.68$ (d, $J=7.6 \mathrm{~Hz}, 1 \mathrm{H}), 5.38$ (s, $1 \mathrm{H}), 4.77$ (d, $J=11.6$ Hz, 1 H), 4.73 - 4.64 (m, 4 H), 3.05 (s, br, 1 H), 1.56 (s, 3 H).

Partial ${ }^{13} \mathrm{C}$ NMR $\left(\mathrm{CDCl}_{3}, 100 \mathrm{MHz}\right): \delta 166.5,166.2,166.0,137.7,133.8,133.7,133.3$, $133.2,130.2,130.1,129.9,129.3,128.9,128.7,128.63,128.57,128.54,128.0,127.6$, 127.5, 127.1, 101.5, 101.2, 83.9, 80.7, 79.1, 78.5, 77.7, 77.1, 67.5, 66.2, 65.5, 64.4, 19.5, 15.8 .

HRMS: Calcd for $\mathrm{C}_{27} \mathrm{H}_{26} \mathrm{O}_{7}-\mathrm{H} \mathrm{m} / \mathrm{z} 461.1600$; Found 461.1622 .

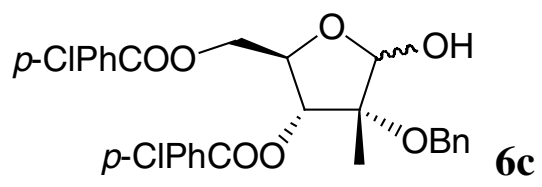

${ }^{1} \mathrm{H}$ NMR $\left(\mathrm{CDCl}_{3}, 400 \mathrm{MHz}\right)$ : The ratio of $\alpha: \beta$ isomers in $\mathrm{CDCl}_{3}$ is about 10 to 7 . For the $\alpha$ isomer: $\delta 8.00-7.90(\mathrm{~m}, 4 \mathrm{H}), 7.43-7.28(\mathrm{~m}, 9 \mathrm{H}), 5.32(\mathrm{~d}, J=5.6 \mathrm{~Hz}, 1 \mathrm{H}), 5.25$ (s, $1 \mathrm{H}), 4.74-4.52(\mathrm{~m}, 5 \mathrm{H}), 4.11(\mathrm{~s}, \mathrm{br}, 1 \mathrm{H}), 1.66(\mathrm{~s}, 3 \mathrm{H})$; for the $\beta$ isomer: $\delta 8.00-$ $7.90(\mathrm{~m}, 4 \mathrm{H}), 7.43-7.28(\mathrm{~m}, 9 \mathrm{H}), 5.66(\mathrm{~d}, J=7.6 \mathrm{~Hz}, 1 \mathrm{H}), 5.42(\mathrm{~s}, 1 \mathrm{H}), 4.76(\mathrm{~d}, J=$ $12.0 \mathrm{~Hz}, 1 \mathrm{H}), 4.74-4.52$ (m, $4 \mathrm{H}), 4.30$ (br s, $1 \mathrm{H}), 1.58$ (s, $3 \mathrm{H})$. 
Partial ${ }^{13} \mathrm{C}$ NMR ( $\left.\mathrm{CDCl}_{3}, 100 \mathrm{MHz}\right): \delta 165.7,165.6,165.4,165.2,140.4,140.3,139.9$, $139.8,137.6,131.5,131.4,131.3,129.10,129.07,129.0,128.9,128.7,128.6,128.3$, 128.1, 128.0, 127.7, 127.6, 127.4, 127.0, 101.4, 101.0, 83.8, 80.7, 79.0, 78.3, 77.9, 77.3, 67.5, 66.2, 65.6, 64.6, 19.6,15.7.

HRMS: Calcd for $\mathrm{C}_{27} \mathrm{H}_{24} \mathrm{Cl}_{2} \mathrm{O}_{7}-\mathrm{H} \mathrm{m} / \mathrm{z}$ 529.0821; Found 529.0815.

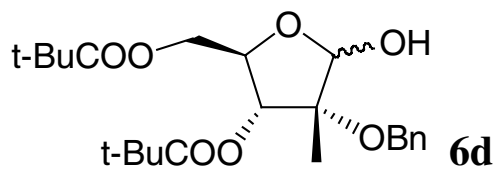

${ }^{1} \mathrm{H} \mathrm{NMR}\left(\mathrm{CDCl}_{3}, 600 \mathrm{MHz}\right)$ : The ratio of $\alpha: \beta$ isomers in $\mathrm{CDCl}_{3}$ is about 4 to 1 . For the $\alpha$ isomer: $\delta 7.37-7.31(\mathrm{~m}, 5 \mathrm{H}), 5.13(\mathrm{~d}, J=7.9 \mathrm{~Hz}, 1 \mathrm{H}), 4.97(\mathrm{~d}, J=5.7 \mathrm{~Hz}, 1 \mathrm{H}), 4.67$ $(\mathrm{d}, J=10.6 \mathrm{~Hz}, 1 \mathrm{H}), 4.55(\mathrm{~d}, J=10.6 \mathrm{~Hz}, 1 \mathrm{H}), 4.38(\mathrm{~m}, 1 \mathrm{H}), 4.27(\mathrm{dd}, J=12.1,3.8$ Hz, $1 \mathrm{H}), 4.19$ (dd, $J=12.1,4.9,1 \mathrm{H}), 3.95(\mathrm{~d}, J=8.3 \mathrm{~Hz}, 1 \mathrm{H}), 1.55$ (s, $3 \mathrm{H}), 1.23$ (s, 9 $\mathrm{H}), 1.13(\mathrm{~s}, 9 \mathrm{H})$; for the $\beta$ isomer (selected data) : $\delta 5.27$ (br s, $1 \mathrm{H}), 5.25(\mathrm{~d}, J=7.6 \mathrm{~Hz}$, $1 \mathrm{H}), 4.71(\mathrm{~d}, J=11.7 \mathrm{~Hz}, 1 \mathrm{H}), 4.60(\mathrm{~d}, J=11.7 \mathrm{~Hz}, 1 \mathrm{H}), 4.34(\mathrm{~m}, 1 \mathrm{H}), 2.78(\mathrm{br} \mathrm{s}, 1$ $\mathrm{H}), 1.45(\mathrm{~s}, 3 \mathrm{H})$.

${ }^{13} \mathrm{C}$ NMR $\left(\mathrm{CDCl}_{3}, 150 \mathrm{MHz}\right): \delta$ ( $\alpha$ isomer) $178.15,177.48,137.49,128.47,127.93$, $127.53,100.86,80.16,78.96,75.88,67.06,63.42,38.84,38.69,27.19,26.99,19.20$.

HRMS: Calcd for $\mathrm{C}_{23} \mathrm{H}_{34} \mathrm{O}_{7}-\mathrm{H} \mathrm{m} / \mathrm{z}$ 421.2226; Found 421.2206.

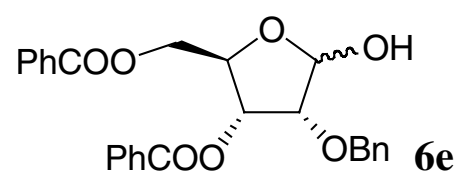

${ }^{1} \mathrm{H}$ NMR $\left(\mathrm{CDCl}_{3}, 600 \mathrm{MHz}\right)$ : The initial ratio of $\alpha: \beta$ in $\mathrm{CDCl}_{3}$, was 2:3. Aging in $\mathrm{CDCl}_{3}$ resulted in a $\alpha: \beta$ ratio of 5:3. For the $\alpha$ isomer: $\delta 8.07(\mathrm{~m}, 2 \mathrm{H}), 7.97(\mathrm{~m}, 2 \mathrm{H})$, $7.61(\mathrm{~m}, 1 \mathrm{H}), 7.56(\mathrm{~m}, 1 \mathrm{H}), 7.47(\mathrm{~m}, 2 \mathrm{H}), 7.40(\mathrm{~m}, 2 \mathrm{H}), 7.29-7.22$ (overlapping $\mathrm{m}, 5 \mathrm{H}$ ), $5.50(\mathrm{dd}, J=8.7,4.2 \mathrm{~Hz}, 1 \mathrm{H}), 5.45(\mathrm{dd}, J=6.0,4.9 \mathrm{~Hz}, 1 \mathrm{H}), 4.70(\mathrm{~d}, J=11.7 \mathrm{~Hz}, 1 \mathrm{H})$, $4.68(\mathrm{~m}, 1 \mathrm{H}), 4.64(\mathrm{~d}, J=11.7 \mathrm{~Hz}, 1 \mathrm{H}), 4.59(\mathrm{dd}, J=12.1,3.8 \mathrm{~Hz}, 1 \mathrm{H}), 4.49(\mathrm{dd}, J=$ $12.1,4.5 \mathrm{~Hz}, 1 \mathrm{H}), 4.27(\mathrm{dd}, J=6.0,4.5 \mathrm{~Hz}, 1 \mathrm{H}), 3.87(\mathrm{~d}, J=8.7 \mathrm{~Hz}, 1 \mathrm{H})$; for the $\beta$ 
isomer: $\delta 8.08(\mathrm{~m}, 2 \mathrm{H}), 8.04(\mathrm{~m}, 2 \mathrm{H}), 7.60(\mathrm{~m}, 1 \mathrm{H}), 7.54(\mathrm{~m}, 1 \mathrm{H}), 7.45(\mathrm{~m}, 2 \mathrm{H}), 7.39(\mathrm{~m}$, 2H), 7.29-7.22 (overlapping m, 5H), 5.59 (dd, $J=6.4,4.9 \mathrm{~Hz}, 1 \mathrm{H}$ ), 5.48 (dd, $J=3.4,1.5$ Hz, 1H), 4.69 (obscured m, 1H), 4.4 (s, 2H), 4.63, (dd, $J=12.1,4.2 \mathrm{~Hz}, 1 \mathrm{H}), 4.58$ (dd, $J$ $=12.1,5.3 \mathrm{~Hz}, 1 \mathrm{H}), 4.29(\mathrm{dd}, J=4.9,1.5 \mathrm{~Hz}, 1 \mathrm{H}, 3.01(\mathrm{~d}, J=3.4 \mathrm{~Hz}, 1 \mathrm{H})$.

${ }^{13} \mathrm{C} \mathrm{NMR}\left(\mathrm{CDCl}_{3}, 150 \mathrm{MHz}\right): \delta 166.4,166.1,165.9,165.9,137.4,136.6,133.6,133.4$, 133.2, 133.1, 129.9, 129.8, 129.7, 129.6, 129.6, 129.4, 129.2, 128.6, 128.5, 128.41, $128.39,128.34,128.29,128.0,127.8,127.7,100.9,96.1,81.4,79.0,78.5,76.6,73.8$, $73.5,73.1,72.2,65.0,64.1$.

HRMS: Calcd for $\mathrm{C}_{26} \mathrm{H}_{24} \mathrm{O}_{7}-\mathrm{H} \mathrm{m} / \mathrm{z}$ 447.1444; Found 447.1455.

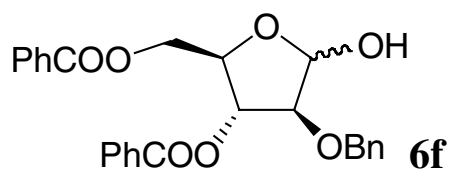

${ }^{1} \mathrm{H}$ NMR $\left(\mathrm{CDCl}_{3}, 400 \mathrm{MHz}\right)$ : The ratio of $\alpha: \beta$ isomers in $\mathrm{CDCl}_{3}$ is about 1 to 2.5. For the $\alpha$ isomer: $\delta 8.06(\mathrm{~m}, 4 \mathrm{H}), 7.48-7.30(\mathrm{~m}, 11 \mathrm{H}), 5.61(\mathrm{~d}, J=2.4 \mathrm{~Hz}, 1 \mathrm{H}), 5.48(\mathrm{dd}, J$ $=0.8,3.6 \mathrm{~Hz}, 1 \mathrm{H}), 4.79$ (d, $J=12.0 \mathrm{~Hz}, 1 \mathrm{H}), 4.74-4.66$ (m, $3 \mathrm{H}), 4.59$ (dd, $J=5.6$, $11.6 \mathrm{~Hz}, 1 \mathrm{H}), 4.21(\mathrm{~s}, 1 \mathrm{H}), 2.90(\mathrm{~d}, J=3.6 \mathrm{~Hz}, 1 \mathrm{H})$; for the $\beta$ isomer: $\delta 8.05(\mathrm{~m}, 4 \mathrm{H})$, $7.62-7.30$ (m, $11 \mathrm{H}), 5.53$ (m, 2 H), 4.89 (d, $J=11.7 \mathrm{~Hz}, 1 \mathrm{H}), 4.75$ (d, $J=11.7 \mathrm{~Hz}, 1$ H), $4.74-4.64$ (m, $2 \mathrm{H}), 4.38$ (ddd, $J=2.9,5.2,8.1 \mathrm{~Hz}, 1 \mathrm{H}$ ), 4.13 (dd, $J=2.2,3.7 \mathrm{~Hz}, 1$ $\mathrm{H}), 3.97(\mathrm{~d}, J=9.5 \mathrm{~Hz}, 1 \mathrm{H})$.

Partial ${ }^{13} \mathrm{C}$ NMR $\left(\mathrm{CDCl}_{3}, 100 \mathrm{MHz}\right): \delta 166.54,166.08,137.45,133.84,133.72,133.28$, 133.20, 130.04, 130.00, 128.88, 128.76, 128.73, 128.56, 128.51, 128.36, 128.15, 128.04, 101.93, 97.45, 87.49, 81.65, 81.41, 79.33, 78.36, 73.12, 72.32, 65.30, 64.63.

HRMS: Calcd for $\mathrm{C}_{26} \mathrm{H}_{24} \mathrm{O}_{7}-\mathrm{H} \mathrm{m} / \mathrm{z}$ 447.1444; Found 447.1455.

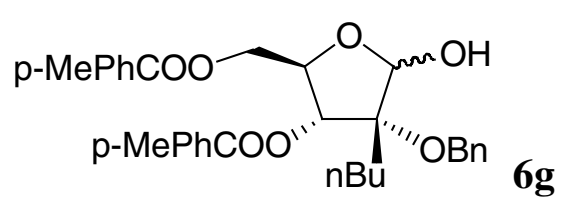


${ }^{1} \mathrm{H} \mathrm{NMR}\left(\mathrm{CDCl}_{3}, 400 \mathrm{MHz}\right)$ : The ratio of $\alpha: \beta$ isomers in $\mathrm{CDCl}_{3}$ is about 1 to $1 . \delta 7.96-$ $7.87(\mathrm{~m}, 8 \mathrm{H}), 7.42-7.14(\mathrm{~m}, 18 \mathrm{H}), 5.76(\mathrm{~d}, J=7.3 \mathrm{~Hz}, 1 \mathrm{H}), 5.43(\mathrm{~d}, J=5.9 \mathrm{~Hz}, 1 \mathrm{H})$, 5.39 (s, $1 \mathrm{H}), 5.35(\mathrm{~s}, 1 \mathrm{H}), 4.92(\mathrm{~d}, J=11.0 \mathrm{~Hz}, 1 \mathrm{H}), 4.74(\mathrm{~d}, J=11.0 \mathrm{~Hz}, 1 \mathrm{H}), 4.70-$ 4.49 (m, 9 H), 4.26 (s, br, 1 H), 3.78 (s, br, 1 H), 2.42 (s, 6 H), 2.41 (s, 3 H), 2.37 (s, 3 H), $2.06-1.79$ (m, 4 H), $1.58-1.27$ (m, 8 H), 0.94 (t, $J=7.3 \mathrm{~Hz}, 3 \mathrm{H}), 0.89$ (t, $J=7.3 \mathrm{~Hz}, 3$ $\mathrm{H})$.

Partial ${ }^{13} \mathrm{C}$ NMR $\left(\mathrm{CDCl}_{3}, 100 \mathrm{MHz}\right): \delta 166.8,166.5,166.0,165.9,144.5,144.4,143.9$, 143.8, 137.7, 130.14, 130.08 129.9, 129.4, 129.22, 129.18, 128.6, 128.53, 128.48, 127.9, $127.6,127.5,127.2,127.0,126.5,101.4,99.7,85.8,82.8,79.1,79.0,77.7,75.7,74.3$, 66.6, 66.0, 65.6, 64.2, 31.7, 29.6, 25.4, 25.3, 23.3, 23.1, 21.82, 21.79, 14.1 .

HRMS: Calcd for $\mathrm{C}_{32} \mathrm{H}_{36} \mathrm{O}_{7}-\mathrm{H} \mathrm{m} / \mathrm{z}$ 531.2383; Found 531.2388.

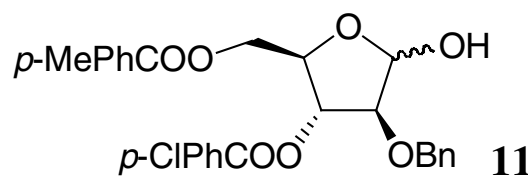

${ }^{1} \mathrm{H}$ NMR $\left(\mathrm{CDCl}_{3}, 400 \mathrm{MHz}\right)$ : The ratio of $\alpha: \beta$ isomers in $\mathrm{CDCl}_{3}$ is about 5 to 2 . For the $\alpha$ isomer: $\delta 7.98(\mathrm{~d}, J=8.5 \mathrm{~Hz}, 2 \mathrm{H}), 7.95(\mathrm{~d}, J=8.1 \mathrm{~Hz}, 2 \mathrm{H}), 7.43(\mathrm{~d}, J=8.5 \mathrm{~Hz}, 2 \mathrm{H})$, 7.34 (m, 5 H), 7.11 (d, $J=8.1 \mathrm{~Hz}, 2 \mathrm{H}), 5.61(\mathrm{~s}, 1 \mathrm{H}), 5.45$ (d, $J=3.3 \mathrm{~Hz}, 1 \mathrm{H}), 4.78$ (d, $J$ $=11.6 \mathrm{~Hz}, 1 \mathrm{H}), 4.69(\mathrm{~m}, 1 \mathrm{H}), 4.68(\mathrm{~d}, J=11.6 \mathrm{~Hz}, 1 \mathrm{H}), 4.65(\mathrm{dd}, J=4.0,11.6 \mathrm{~Hz}, 1$ H), $4.56(\mathrm{dd}, J=5.6,11.6 \mathrm{~Hz}, 1 \mathrm{H}), 4.18(\mathrm{~s}, 1 \mathrm{H}), 2.94(\mathrm{~s}, \mathrm{br}, 1 \mathrm{H}), 2.38(\mathrm{~s}, 3 \mathrm{H})$; for the $\beta$ isomer: $\delta 7.94(\mathrm{~d}, J=8.0 \mathrm{~Hz}, 4 \mathrm{H}), 7.45(\mathrm{~d}, J=8.0,2 \mathrm{H}), 7.34(\mathrm{~m}, 5 \mathrm{H}), 7.18(\mathrm{~d}, J=8.0$ $\mathrm{Hz}, 1 \mathrm{H}), 5.51(\mathrm{~m}, 2 \mathrm{H}), 4.86(\mathrm{~d}, J=11.9 \mathrm{~Hz}, 1 \mathrm{H}), 4.75(\mathrm{~d}, J=11.9 \mathrm{~Hz}, 1 \mathrm{H}), 4.69$ (m, 1 H), $4.60(\mathrm{~m}, 1 \mathrm{H}), 4.35$ (ddd, $J=3.4,3.4,6.0 \mathrm{~Hz}, 1 \mathrm{H}), 4.11(\mathrm{dd}, J=3.3,3.3,1 \mathrm{H}), 3.98$ (br d, $J=8.7 \mathrm{~Hz}, 1 \mathrm{H}), 2.39$ (s, $3 \mathrm{H})$.

${ }^{13} \mathrm{C} \mathrm{NMR}\left(\mathrm{CDCl}_{3}, 100 \mathrm{MHz}\right): \delta$ 166.6, 165.2, 165.1, 144.0, 143.9, 140.4, 140.2, 137.4, 136.8, 131.42, 131.36, 130.1, 130.0, 129.3, 129.2, 129.12, 129.07, 128.9, 128.7, 128.6, $128.4,128.1,128.04,127.79,127.2,101.9,97.4,87.4,81.6,81.4,79.3,78.6,77.9,73.1$, 72.3, 65.1, 64.4, 21.8.

HRMS: Calcd for $\mathrm{C}_{27} \mathrm{H}_{25} \mathrm{ClO}_{7}-\mathrm{H} \mathrm{m} / \mathrm{z}$ 495.1211; Found 495.1229. 


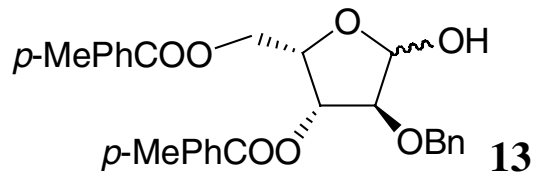

${ }^{1} \mathrm{H}$ NMR $\left(\mathrm{CDCl}_{3}, 400 \mathrm{MHz}\right)$ : The ratio of $\alpha: \beta$ isomers in $\mathrm{CDCl}_{3}$ is about 1 to 2 . For the $\beta$ isomer: $\delta 7.89(\mathrm{~m}, 4 \mathrm{H}), 7.40-7.30(\mathrm{~m}, 5 \mathrm{H}), 7.26-7.19(\mathrm{~m}, 4 \mathrm{H}), 5.64(\mathrm{dd}, J=1.6$, $4.0 \mathrm{~Hz}, 1 \mathrm{H}), 5.60$ (s, br, $1 \mathrm{H}), 4.95$ (d, $J=11.6 \mathrm{~Hz}, 1 \mathrm{H}), 4.78$ (m, $2 \mathrm{H}), 4.55$ (d, $J=5.8$ Hz, $2 \mathrm{H}), 4.12$ (dd, J = 1.6, $4.0 \mathrm{~Hz}, 1 \mathrm{H}), 4.54$ (s, br, $1 \mathrm{H}), 2.42$ (s, $3 \mathrm{H}), 2.41$ (s, $3 \mathrm{H})$; for the $\alpha$ isomer: $\delta 7.90(\mathrm{~m}, 4 \mathrm{H}), 7.40-7.30(\mathrm{~m}, 5 \mathrm{H}), 7.26-7.19(\mathrm{~m}, 4 \mathrm{H}), 5.66(\mathrm{dd}, J=$ $1.3,4.8 \mathrm{~Hz}, 1 \mathrm{H}), 5.48(\mathrm{~s}, 1 \mathrm{H}), 4.86-4.69(\mathrm{~m}, 4 \mathrm{H}), 4.57$ (dd, $J=5.2,11.6 \mathrm{~Hz}, 1 \mathrm{H}), 4.16$ (s, $1 \mathrm{H}), 3.00$ (s, br, $1 \mathrm{H}), 2.41$ (s, $3 \mathrm{H}), 2.40$ (s, $3 \mathrm{H})$.

${ }^{13} \mathrm{C} \mathrm{NMR}\left(\mathrm{CDCl}_{3}, 100 \mathrm{MHz}\right): \delta 166.4,165.9,144.8,144.0,136.7,130.00,129.97,129.5$, $129.3,128.9,128.7,128.6,128.3,128.2,128.0,127.2,126.5,102.2,96.8,87.1,81.2$, 78.9, 76.4, 75.8, 75.7, 73.5, 72.4, 63.8, 62.7, 21.91, 21.86.

HRMS: Calcd for $\mathrm{C}_{28} \mathrm{H}_{28} \mathrm{O}_{7}-\mathrm{H} \mathrm{m} / \mathrm{z}$ 475.1757; Found 475.1774.

\section{Preparation of 8}

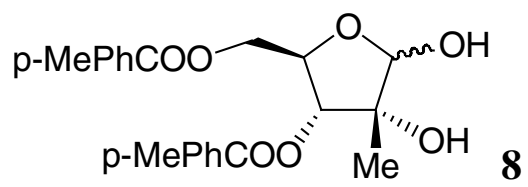

To a iPrOAc solution of $\mathbf{6 a}$ from above procedure was added Charcoal (Darco G60, $500 \mathrm{~g})$. The slurry was aged for $1 \mathrm{~h}$ at ambient temperature. Then, the slurry was filtered through Solka Floc (10 g) to remove charcoal. The organic phase was diluted with iPrOAc to $500 \mathrm{~mL}$. Pd/C $(1.5 \mathrm{~kg}, 10 \%)$ was charged. The reaction mixture was subjected to hydrogenolysis $\left(45 \mathrm{psi}_{2}, 50-60{ }^{\circ} \mathrm{C}\right.$ ) for $35 \mathrm{~h}$. The reaction mixture was cooled to ambient temperature and was filtered through Solka Flok. The iPrOAc solution was concentrated to about $135 \mathrm{~mL}$. The solution was heated to $50{ }^{\circ} \mathrm{C}$ and $90 \mathrm{~mL}$ of heptane was added in one portion. The solution was seeded with $2.5 \mathrm{~g}$ of $\mathbf{8}$ and allowed to age for $2 \mathrm{~h}$ at $50{ }^{\circ} \mathrm{C}$. Heptane $(270 \mathrm{~mL})$ was added dropwise over $2.5 \mathrm{~h}$. The slurry was aged at $50{ }^{\circ} \mathrm{C}$ for $1 \mathrm{~h}$, then cooled to ambient temperature and aged for $2 \mathrm{~h}$ before filtration. The wet cake was displacement washed with $90 \mathrm{~mL}$ of $20 \%$ iPrOAc/heptane followed by 120 
$\mathrm{mL} \times 2$ of $10 \%$ iPrOAc/heptane. The solids were suction dried under a $\mathrm{N}_{2}$ atmosphere at ambient temperature to give $4.54 \mathrm{~kg}$ of $8.90 \mathrm{wt} \% .66 \%$ isolated for overall five-step through process.

${ }^{1} \mathrm{H} \mathrm{NMR}\left(\mathrm{CDCl}_{3}, 400 \mathrm{MHz}\right)$ : The ratio of $\alpha: \beta$ isomers in $\mathrm{CDCl}_{3}$ is about 5 to 1 . For the $\alpha$ isomer: $\delta 7.95-7.90(\mathrm{~m}, 4 \mathrm{H}), 7.26(\mathrm{~d}, J=8.0 \mathrm{~Hz}, 2 \mathrm{H}), 7.17(\mathrm{~d}, J=8.0 \mathrm{~Hz}, 2 \mathrm{H}), 5.53$ $(\mathrm{d}, J=7.2 \mathrm{~Hz}, 1 \mathrm{H}), 5.22(\mathrm{~d}, J=2.8 \mathrm{~Hz}, 1 \mathrm{H}), 4.65-4.49(\mathrm{~m}, 3 \mathrm{H}), 3.08(\mathrm{~d}, J=3.2 \mathrm{~Hz}, 1$ $\mathrm{H}), 2.44(\mathrm{~s}, 3 \mathrm{H}), 2.38(\mathrm{~s}, 3 \mathrm{H}), 2.26(\mathrm{~s}, 1 \mathrm{H}), 1.44(\mathrm{~s}, 3 \mathrm{H})$; for the $\beta$ isomer: $\delta 7.95-7.90$ (m, 4H), $7.27(\mathrm{~d}, J=8.0 \mathrm{~Hz}, 2 \mathrm{H}), 7.22(\mathrm{~d}, J=8.0 \mathrm{~Hz}, 2 \mathrm{H}), 5.16(\mathrm{~d}, J=5.6 \mathrm{~Hz}, 1 \mathrm{H})$, $5.12(\mathrm{~d}, J=5.6 \mathrm{~Hz}, 1 \mathrm{H}), 4.66-4.49(\mathrm{~m}, 3 \mathrm{H}), 3.54(\mathrm{~d}, J=5.6 \mathrm{~Hz}, 1 \mathrm{H}), 2.91(\mathrm{~s}, 1 \mathrm{H})$, $2.43(\mathrm{~s}, 3 \mathrm{H}), 2.40$ (s, $3 \mathrm{H}), 1.44$ (s, $3 \mathrm{H})$.

${ }^{13} \mathrm{C} \mathrm{NMR}\left(\mathrm{CDCl}_{3}, 100 \mathrm{MHz}\right): \delta 166.6,166.3,165.9,165.7,144.6,144.3,143.8,143.7$, 129.9, 129.7, 129.3, 129.2, 129.1, 129.0, 127.0, 126.9, 126.4, 126.2, 102.9, 100.8, 79.8, 79.2, 78.7, 76.9, 76.5, 76.4, 65.5, 64.0, 23.5, 21.7, 21.6, 20.0.

HRMS: Calcd for $\mathrm{C}_{22} \mathrm{H}_{24} \mathrm{O}_{7}-\mathrm{H} \mathrm{m} / \mathrm{z}$ 399.1444; Found 399.1453. 